\title{
A quasi-random spanning tree model for the early river network
}

\author{
S. S. Manna ${ }^{1,2, \text {, }}$ and B. Subramanian ${ }^{2}$ \\ ${ }^{1}$ Höchstleistungsrechenzentrum des Forschungszentrum, D-52425 Jülich , Germany \\ ${ }^{2}$ Department of Physics, Indian Institute of Technology, Bombay 400076, India
}

\begin{abstract}
We consider a model for the formation of a river network in which erosion process plays a role only at the initial stage. Once a global connectivity is achieved, no further evolution takes place. In spite of this, the network reproduces approximately most of the empirical statistical results of natural river network. It is observed that the resulting network is a spanning tree graph and therefore this process could be looked upon as a new algorithm for the generation of spanning tree graphs in which different configurations occur quasi-randomly. A new loop-less percolation model is also defined at an intermediate stage of evolution of the river network.
\end{abstract}

PACS numbers : 64.60.Ht, 92.40.Gc, 05.40.+j, 05.20.-y

\footnotetext{
*manna@niharika.phy.iitb.ernet.in
} 
A river network consists of a main river accompanied by a hierarchy of side streams of decreasing lengths and flow capacities. Ignoring the ground absorption and evaporation, the network drains out the whole amount of rain water dropped uniformly on every small piece of land in the river basin and therefore necessarily spans the whole drainage area. In addition, though it is quite common that two rivers join together, it is hardly observed that a river bifurcates into two smaller streams (ignoring small delta islands) because of the fact that water flows in the direction of steepest descent. Therefore the general structure of a river network is like a tree on which two points are connected by a distinct path. Due to these properties, a river network qualifies to be described by a spanning tree graph, the loop-less graph which covers all nodes, a well known example in graph theory [1]. The aim of this paper is to propose a quasi-random spanning tree model for the formation of a river network from the very early stage.

The geometrical structure of the network considering different streams as linear segments has been of considerable interest for a long time. Quite commonly different rivers are classified according to Strahler's ordering procedure [2]. In this recursive ordering scheme, two streams of orders $n_{1}$ and $n_{2}$ meet to produce a stream of order $n$ as :

$$
\text { if } \quad n_{1} \neq n_{2} \quad \text { then } n=\max \left(n_{1}, n_{2}\right) ; \quad \text { else, } \quad n=n_{1}+1
$$

where the streams which start from sources are assigned order $n=1$. Horton empirically observed that the average number and length of rivers of different orders in a network follow geometric series with approximately constant bifurcation $r_{b}$ and the length ratio $r_{l}$ [3]. Mandelbrot suggested that the river network might be a self-similar fractal with fractal dimension $d_{r n}=2$ because of the spanning nature of the network [4]. Later, fractal dimension of the individual rivers $d_{c}$ are related to the Horton's ratios by $[5,6]$

$$
d_{r n}=d_{c} \frac{\log r_{b}}{\log r_{l}} .
$$

The mean annual discharge at any link or its surrogate variable, the cumulative area contributing to the link follows a power law probability distribution as, $P(a) \sim a^{-\tau_{a}}$ [7]. The 
length $l$ of a typical stream of certain order also follows a power law distribution $P(l) \sim l^{-\tau_{l}}$ [8]. Another empirical result is that the average length $\left\langle l_{\Omega}\right\rangle$ of the river with maximum order $\Omega$ varies with the basin area $a$ as $l_{\Omega} \sim a^{\alpha}[9]$.

Scheidegger proposed a lattice model of directed river network defined on a slope [10]. This model was shown to be identical to the one dimensional random particle aggregation model [11]. More recently, following the idea of Self-Organized Criticality [12], a number of river network models are proposed which successfully produce spatial scale invariance in the self-organized critical states [13].

The problem of spanning tree graphs is well known in statistical physics. Kirchhoff related the spanning tree graphs to the problem of determining the effective resistance between two nodes of a resistor network [14]. Fortuin and Kasteleyn showed that it is related to the $q \rightarrow 0$ limit of the $q$-state Potts model [15]. Recently the correspondence between the spanning tree graphs and the steady state configurations of the Abelian sandpile model in the Self-Organized Criticality [16] is established. In the case of random spanning tree problem all possible tree configurations occur with equal probability and this model is very well studied. We compare the results of our model with those of random spanning trees and conclude that our model belongs to a new universality class.

The process of erosion is the underlying mechanism for the evolution of a river network. Erosion takes place during the flow of streams which modifies the river beds and therefore causes changes in the flow pattern. We consider here the evolution of a network to its full connected form starting from the very initial stage of isolated lakes.

Continuously variable heights with uniform random distributions are assigned to all sites of a square lattice. We first assume that rain falls only along the bonds of the lattice and flows downwards on the slope along the bond. Due to this unidirectional flow, intensive erosion process takes place which reduces the slope along the bond. We assume that this reduction of slope due to the erosion is a very slow process since huge amounts of sediments are transported from one place to the other. When the slope decreases water gets accumulated in different places along the bond and finally when the slope is very small a little further rain 
fall in a very short time floods the whole length of the bond, forming a lake of the smallest size of only one bond. We assume that this transition time is much smaller than the time required for the whole erosion process.

Rain falls simultaneously along all the bonds of the lattice, the erosion process takes place in parallel and lattice bonds become lakes one after another sequentially occuring with uniform probability. Several one bond lakes join together and form bigger lakes. We assume that all sites of one lake have approximately equal heights and since the water in a lake is stagnant, there is no significant erosion takes place to change their heights further.

For the case of a bond which does not belong to any lake but the adjacent two sites belong to the same lake, situation is different. Since the sites are approximately of same height, there is no significant height gradient along the bond and the erosion process will not be fast enough to equalize the level of the bond with the sites. Therefore this bond will never be included into the lake forbidding the possiblity of loop formation.

The role of a lake is to store rain water in the initial stage, untill it gets connected to a flowing river when it also starts flowing and becomes part of the river network. The first site on the boundary of the lattice which becomes the member of a particular lake is connected to the ocean outside. This creates a net directed flow in every bond of this lake which form a small cluster of rivers. In a similar manner all other lakes also eventually become connected among themselves and to this small cluster of rivers and therefore will start flowing. Positive slopes against the flow are created due to erosion along all the bonds which completes the formation of the river network.

A random list of all bonds $B$ of the lattice is generated from an ordered sequence by large number of random pair interchanges. Here the computational effort increases linearly with $B$ to obtain a most uncorrelated configuration, compared to $B \log B$ in the Broder's algorithm for generating the random spanning trees [17].

Numbers are called sequentially from this random list and corresponding bonds on the lattice are tested for the lake formation. A bond is allowed to be a lake if it is the smallest lake, becomes part of the bigger lake or joins two distinct lakes. A bond is forbidden 
to be occupied by a lake if it connects two sites of the same lake. We use the Hoshen and Kopelman's algorithm for cluster numbering in percolation theory [18] for identifying different lakes and to restrict loop formations. Finally a single connected network in the form of a spanning tree graph covers the whole lattice (Fig. 1).

We first exactly calculate the probabilities of occuring for different spanning trees generated from random permutations on a $2 \times 3$ cell and observe that they are non-uniformly distributed (Fig. 2). We conclude that spanning trees obtained from random bond permutations occur with non-uniform probabilities and therefore we call them as 'quasi-random'.

Periodic boundary conditions are used in all four directions of the square lattice and the outlet of the network is chosen at the root of the spanning tree. We first study the connectivity of a randomly chosen site. The average fractions of sites connected to $1,2,3$ and 4 bonds are obtained as $0.30681,0.42698,0.22557$ and 0.04061 , slightly different from their counterparts $0.29454,0.44699,0.22239$ and 0.03608 in random spanning trees [19].

The drainage area at the site $i$ is defined as $a_{i}=\Sigma_{j} w_{i j} a_{j}+1$ where $j$ runs over the nearest neighbour sites and $w_{i j}=1$ if flow direction is from $j$ to $i$, otherwise it is zero. Area values are calculated using a systematic deleting procedure. Leaf sites of the network are the set of sites connected by only one bond, initially unit area values are assigned to them. At the deleting time $t$ all the leaf sites are deleted simultaneously and area values are carried over to the connected sites. This creates a new set of leaf sites to be deleted in the time $t+1$. The root gets an area $L^{2}$. In the figure 3 we plot the probability distribution of the drainage area $P(a)$ for $L=1024$ and obtain a very nice straight line. We estimate $\tau_{a}=1.392 \pm 0.010$ and compare with $11 / 8$ for random spanning trees [19] and to $4 / 3$ of the directed river network model [10,11]. Empirical values of $\tau_{a}$ varies from 1.41 to 1.44 for different river basins [7].

To calculate the stream length distribution $P(l)$ we delete different streams sequentially one after the other. Deletion starts from a leaf site, proceeds along the river and stops when the river meets a higher order river. When all first order rivers are deleted, we get another set of leaf sites all of which correspond to the second order rivers, which are also eventually 
deleted. Using $L=1024$ we get the exponent $\tau_{l}=2.65 \pm 0.03$, to be compared with its empirical value 2.9 [8].

The average length $<l_{\Omega}>$ of the rivers with maximum order number $\Omega$ varies with the whole basin area $L^{2}$ with a power $\alpha=0.636 \pm 0.005$. Empirically one gets $\alpha=0.58 \pm 0.03$ $[9]$.

We also studied the statistics of the longest river flowing into a site. The deleting time of any site is the length of the longest river $l_{m}$ at that site. This length also follows a power law distribution $P\left(l_{m}\right) \sim l_{m}^{-\tau_{m}}$ with $\tau_{m}=1.628 \pm 0.005$. Similarly defined exponent $\alpha_{m}$ in $l_{m} \sim<a>^{\alpha_{m}}$ is obtained as $0.608 \pm 0.005$. This gives a connection between $a$ and $l_{m}$ as $P(a) d a=P\left(l_{m}\right) d l_{m}$ and the scaling relation $\alpha_{m}=\left(\tau_{a}-1\right) /\left(\tau_{m}-1\right)$ gives approximately the same value of $\alpha_{m}$ as measured numerically.

The chemical distance between any two points is defined as the length of the shortest connecting path. Therefore to calculate the fractal dimension of the rivers in our model we calculate the dimension of the chemical paths. A reverse deleting of the network is done from the root of the tree. The deleting time of a site is the length of the river to that site from the root. The probability that an arbitrarily selected site is at a chemical distance $l_{r}$ from the root follows a scaling form $P\left(l_{r}, L\right)=L^{d_{c}} f\left(l_{r} / L^{d_{c}}\right)$. From an excellent data collapse of this distribution data for $L=64,256$ and 1024 we get $d_{c}=1.217$. The scaling function also fits very well to the form $f(x)=a x^{b} \exp \left(-c x^{d}\right)$ where $a=1.30, b=0.59, c=0.60, d=2.62$. We also get another value of $d_{c}=1.222$ by directly calculating the average length of the river $<l_{r}(L)>\sim L^{d_{c}}$. We conclude $d_{c}=1.220 \pm 0.010$ and compare it with the random spanning tree value $5 / 4[20]$.

In figure 4 we plot the average number of rivers $\left\langle N_{n}\right\rangle$ and the average length of the rivers $\left\langle l_{n}>\right.$ for different orders $n$ for a lattice of length $L=1024$. We obtain almost constant value of Horton's bifurcation ratio $r_{b}=4.39$ and the length ratio $r_{l}=2.44$ in the region from $n=2$ to 7 . We compare these values with Shreve's calculation of $r_{b}=4$ and $r_{l}=2$ for the equally weighted river networks [21]. Using the eqn. (2) and the fractal dimension of the rivers $d_{c}=1.220$ we calculate that fractal dimension of the river network 
$d_{r c}=2.02$ which is quite close to its exact value 2 for our quasi-random spanning tree river network.

Finally we consider the situation where rain falls non uniformly or the basin area contains some randomly positioned dry lands using a new percolation model. We randomly throw bonds on the lattice in the same way as before but keep checking if the connectivity is formed between any two opposite sites of the lattice. The moment it is formed we stop further dropping of bonds. We see that $p_{c}(\infty)-p_{c}(L) \sim L^{-1 / \nu}$ where $p_{c}(\infty)=0.4511 \pm$ 0.0005 and $\nu=1.334 \pm 0.005$, which is very close to the value of $\nu=4 / 3$ but the value of $d_{c}=1.119 \pm 0.005$ is distinctly different from $1.1307 \pm 0.0004$ for ordinary percolation [22] Percolation on the Bethe lattices are previously considered by Straley [23].

To summarize, we have considered the formation of a globally connected river network starting from the very early stage of water accumulation in the microscopic lakes. Lakes grow in size and eventually get connected to the ocean when its different branches become rivers. Finally the river network spans the basin. We model the river network by a quasirandom spanning tree belonging to a new universality class. We see that though we have not considered the temporal development of rivers, the first connected network closely reproduces the statistics of the natural river network. We also study a new loop-less perolation model at an intermediate stage of evolution of the river network.

After finishing this work we came to know about the work of Cieplak et. al. [24] who considered disorder-dominated river basins and obtained results similar to us.

We thank D. Dhar for many useful suggestions, H. Kallabis for much help in graphics and A. Giacometti and D. Wolf for the critical reading of the manuscript. 


\section{Figure Captions}

Figure 1 : A typical quasi-random spanning tree configuration for modelling the river network on the $32 \times 32$ lattice. Rivers of order 1(black), 2(yellow), 3(blue), 4(green) and 5(red) are shown. Connection to the ocean is through the site with a circle at the bottom.

Figure 2 : The fifteen distinct spanning tree configurations on a $2 \times 3$ lattice. The nine type (a) configurations occur 360 times and the six type (b) configurations occur 300 times in all the spanning tree configurations generated by exact enumeration of the $7 !=5040$ permutations of the seven bonds of the lattice.

Figure 3 : The probability distribution $P(a)$ of finding an arbitrarily selected site of drainage area $a$ is shown for $L=1024$, which gives $\tau_{a}=1.392$.

Figure 4 : The average number of rivers $\left\langle N_{n}\right\rangle$ (denoted by circles) and the average length of the rivers $\left\langle l_{n}>\right.$ (denoted by crosses) for different order numbers are plotted against $n$. The bifurcation ratio $r_{b}=<N_{n}>/<N_{n+1}>$ and length ratio $r_{l}=<l_{n+1}>$ $/<l_{n}>$ are obtained 4.39 and 2.44 respectively. 


\section{References}

[1] F. Harary, Graph Theory (Addition-Wesley, Reading, MA, 1990).

[2] A. N. Strahler, Trans. Am. Geophys. Union 38, 913 (1957).

[3] R. E. Horton, Geol. Soc. Am. Bull. 56, 275 (1945).

[4] B. B. Mandelbrot, The Fractal Geometry of Nature, W. H. Freeman, New York (1983).

[5] P. La Barbera and R. Rosso, Water Resour. Res. 25, 735 (1989).

[6] D. G. Tarboton, R. L. Bras and I. Rodriguez-Iturbe, Water Resour. Res. 26, 2243 (1990).

[7] I. Rodriguez-Iturbe, E. J. Ijjàsz-Vàsquez, R. L. Bras and D. G. Tarbotton, Water Resour. Res. 28, 1089 (1992).

[8] D. G. Tarboton, R. L. Bras and I. Rodrigues-Iturbe, Water Resour. Res., 24, 1317 (1988).

[9] J. T. Hack, U. S. Geol. Surv. Prof. Pap. 294-B (1957).

[10] A. E. Scheidegger, Bull. I.A.S.H. 12 (1), 15 (1967).

[11] H. Takayasu, Phys. Rev. Lett. 63, 2563 (1989); T. Nagatani, J. Phys. A. 26, L489 (1993).

[12] P. Bak, C. Tang and K. Wiesenfeld, Phys. Rev. lett. 59, 381 (1987).

[13] H. Takayasu and H. Inaoka, Phys. Rev. Lett. 68, 966 (1992); A. Rinaldo, I. Rodriguez-Iturbe, R. Rigon, E. Ijjasz-Vasquez and R. L. Bras, Phys. Rev. Lett. 70, 822 (1993); A. Giacometti, A. Maritan and J. R. Banavar, Phys. Rev. Lett. 75, 577 (1995).

[14] G. Kirchhoff, Ann. Phys. Chem. 72, 497 (1847).

[15] C. M. Fortuin and P. W. Kasteleyn, Physica 57, 536 (1972).

[16] S. N. Majumdar and D. Dhar, Physica A 185, 129 (1992).

[17] A. Z. Broder, in Proceedings of the 30-th Annual IEEE Symposium on Foundations of Computer Science, (IEEE, New York, 1989), p.442.

[18] J. Hoshen and R. Kopelman, Phys. Rev. B., 14, 3428 (1976)

[19] S. S. Manna, D. Dhar and S. N. Majumdar, Phys. Rev. A., 46, 4471 (1992). 
[20] B. Duplantier, J. Stat. Phys. 54, 581 (1989); A. Coniglio, Phys. Rev. Lett. 62, 3054 (1989).

[21] R. L. Shreve, J. Geol. 74, 17 (1966); 77, 397 (1969).

[22] P. Grassberger, J. Phys. A. 25, 5475 (1992).

[23] J. P. Straley, J. Phys. C. 9, 783 (1976).

[24] M. Cieplac, A. Giacometti, A. Maritan, A. Rinaldo, I Rodriguez-Iturbe and J. R. Banavar, unpublished. 


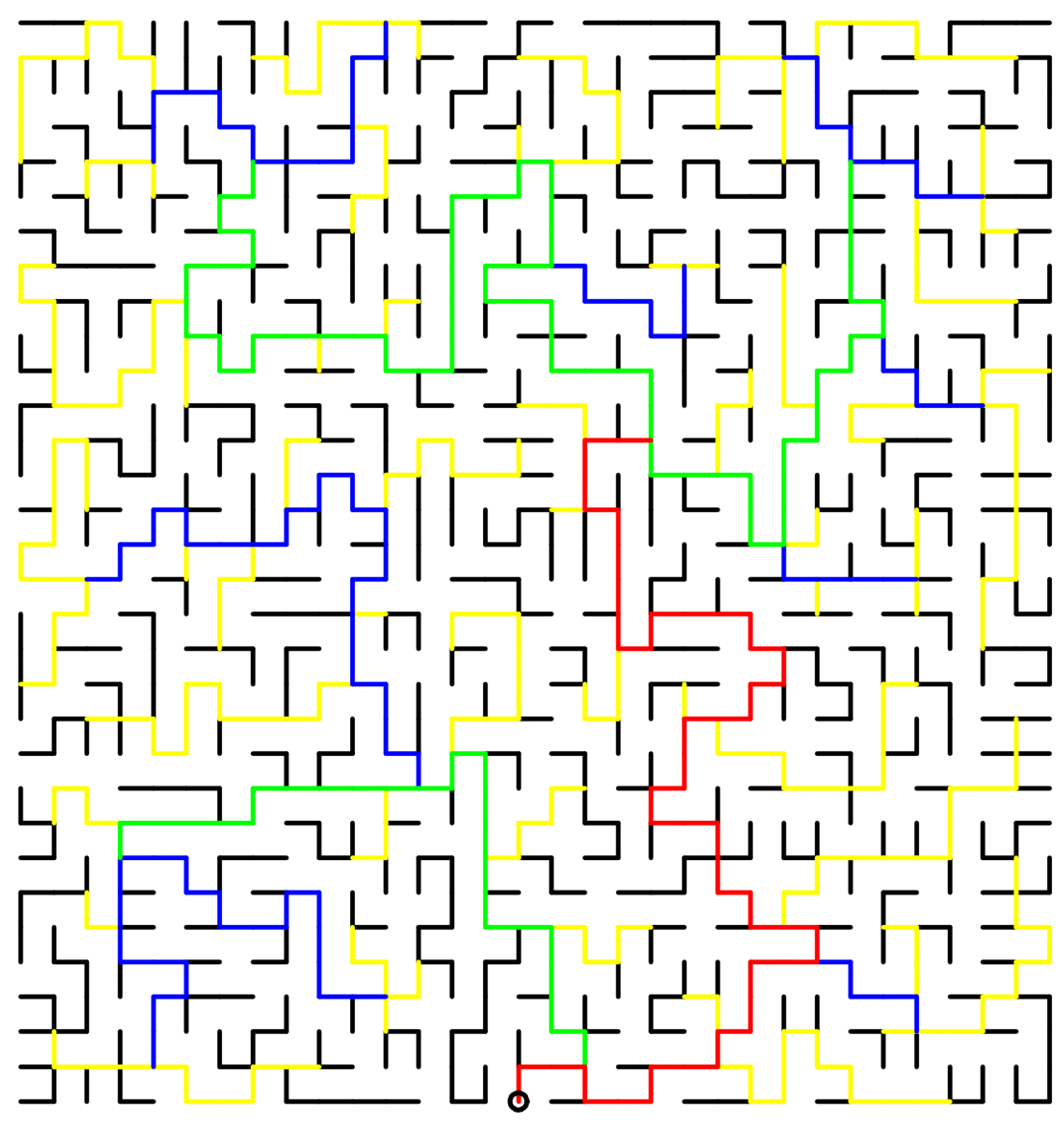

Fig 1 (Manna and Subramanian) 
(a)
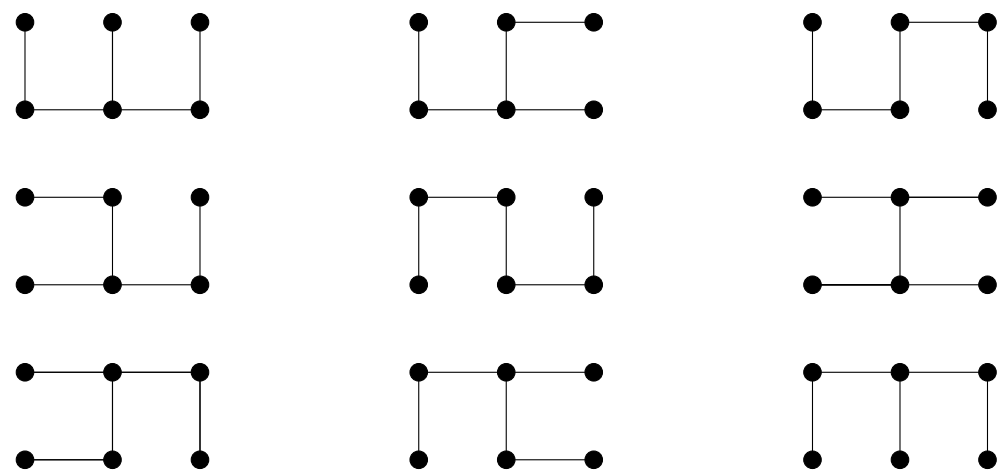

(b)
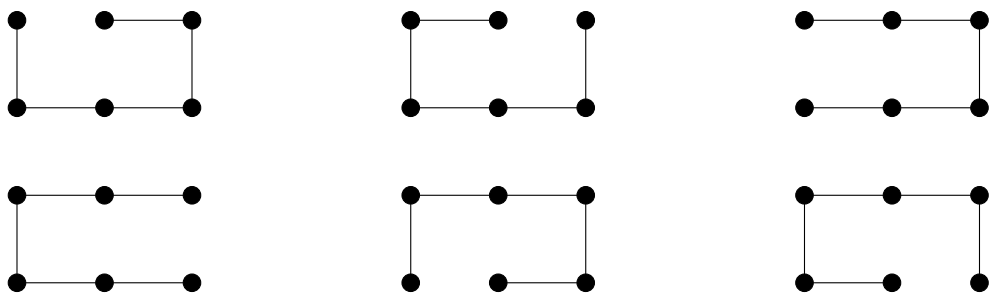

Fig2 (Manna and Subramanian) 
Fig 3 (Manna and Subhramanian)

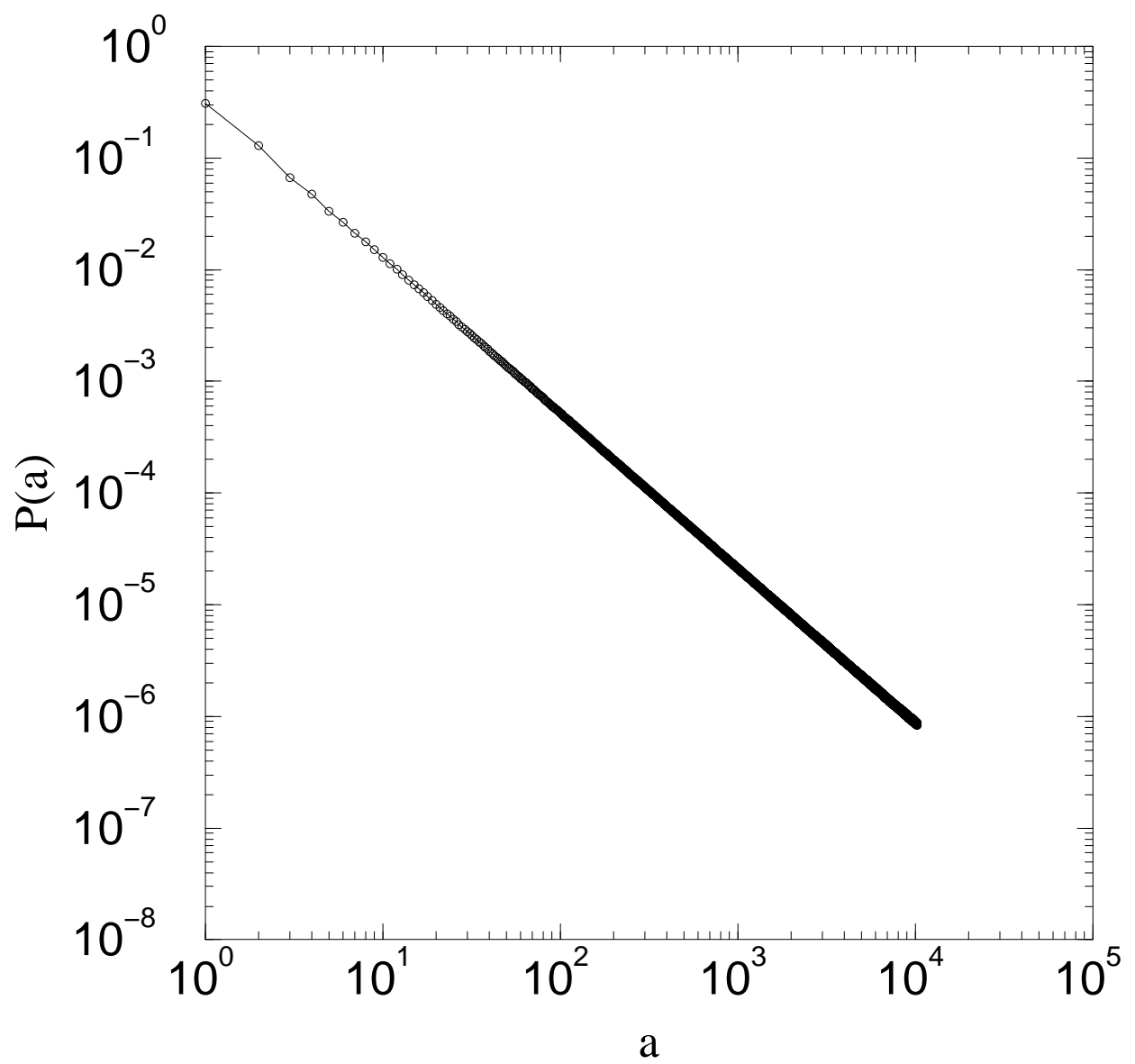


Fig 4 (Manna and Subramanian)

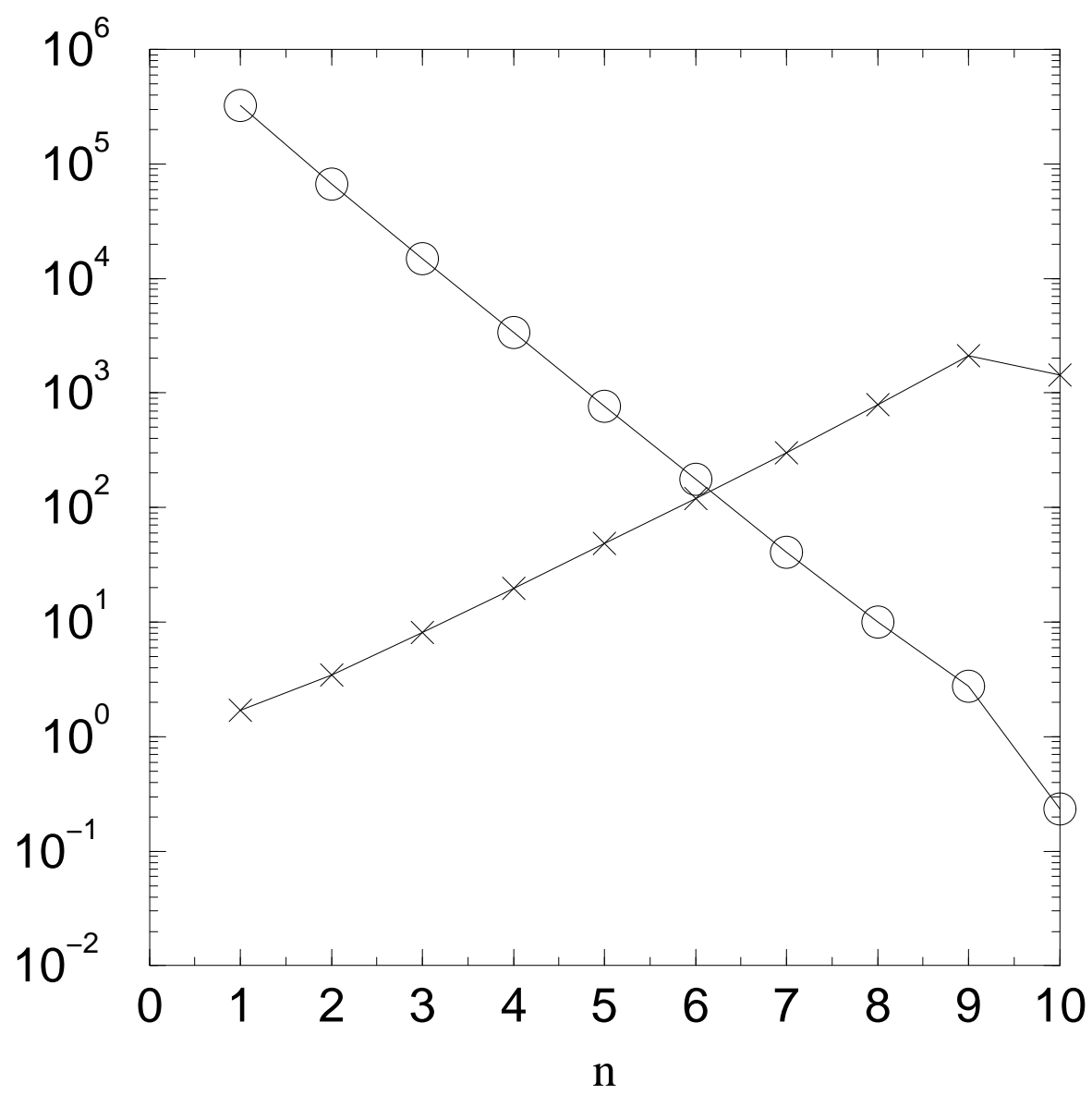

